\title{
ON SOME APPLICATIONS OF THE BOUNDARY CONTROL METHOD TO SPECTRAL ESTIMATION AND INVERSE PROBLEMS
}

\author{
S. A. Avdonin ${ }^{1}$, A. S. Mikhaylov ${ }^{2,3}$, V.S. Mikhaylov ${ }^{2,4}$ \\ ${ }^{1}$ Department of Mathematics and Statistics, University of Alaska, Fairbanks, USA \\ ${ }^{2}$ St. Petersburg Department of V.A. Steklov Institute of Mathematics of the Russian Academy \\ of Sciences, St. Petersburg, Russia \\ ${ }^{3}$ St. Petersburg State University, Faculty of Mathematics and Mechanics, St. Petersburg, Russia \\ ${ }^{4}$ St. Petersburg State University, Faculty of Physics, St. Petersburg, Russia \\ s.avdonin@alaska.edu,mikhaylov@pdmi.ras.ru,vsmikhaylov@pdmi.ras.ru
}

PACS 02.30 Zz, 02.30 Yy, 02.30 Nw

DOI 10.17586/2220-8054-2015-6-1-63-78

We consider applications of the Boundary Control (BC) method to generalized spectral estimation problems and to inverse source problems. We derive the equations of the $\mathrm{BC}$ method for these problems and show that the solvability of these equations crucially depends on the controllability properties of the corresponding dynamical system and properties of the corresponding families of exponentials.

Keywords: spectral estimation problem, boundary control method, identification problem, inverse problem, Schrödinger equation, hyperbolic system.

Received: 5 December 2014

\section{Introduction}

The classical spectral estimation problem consists of recovering the coefficients $a_{n}, \lambda_{k}$, $k=1, \ldots, N, N \in \mathbb{N}$, of a signal

$$
s(t)=\sum_{n=1}^{N} a_{k} e^{\lambda_{k} t}, \quad t \geqslant 0
$$

from the given observations $s(j), j=0, \ldots, 2 N-1$, where the coefficients $a_{k}, \lambda_{k}$ may be arbitrary complex numbers. The literature describing various methods for solving the spectral estimation problem is very extensive: see for example the list of references in [1,2]. In these papers a new approach to this problem was proposed: a signal $s(t)$ was treated as a kernel of a certain convolution operator corresponding to an input-output map for some linear discretetime dynamical system. While the system realized from the input-output map is not unique, the coefficients $a_{n}$ and $\lambda_{n}$ can be determined uniquely using the non-selfadjoint version of the boundary control method [3].

In $[4,8]$, this approach has been generalized to the infinite-dimensional case: more precisely, the problem of the recovering the coefficients $a_{k}, \lambda_{k} \in \mathbb{C}, k \in \mathbf{N}$, of the given signal:

$$
S(t)=\sum_{k=1}^{\infty} a_{k}(t) e^{\lambda_{k} t}, \quad t \in(0,2 T),
$$

from the given data $S \in L_{2}(0,2 T)$ was considered. In [4], the case $a_{k} \in \mathbb{C}$ has been treated, in [8] the case when for each $k, a_{k}(t)=\sum_{i=0}^{L_{k}-1} a_{k}^{i} t^{i}$ are polynomials of the order $L_{k}-1$ with complex valued coefficients $a_{k}^{i}$ was studied. 
Recently, it was observed $[9,15]$ that the results of $[4,8]$ are closely related to the dynamical inverse source problem: let $H$ be a Hilbert space, $A$ be an operator in $H$ with the domain $D(A)$, Y be another Hilbert space, $O: H \supset D(O) \mapsto Y$ be an observation operator (see [18]). Given the dynamical system in $H$ :

$$
\left\{\begin{array}{l}
u_{t}-A u=0, \quad t>0, \\
u(0)=a,
\end{array}\right.
$$

we denote by $u^{a}$ its solution, and by $y(t):=\left(O u^{a}\right)(t)$ the observation (output of this system). The operator that realizes the correspondence $a \mapsto\left(O u^{a}\right)(t)$ is called the observation operator $\mathbb{O}^{T}: H \mapsto L_{2}(0, T ; Y)$. We fix some $T>0$ and assume that $y(t) \in L_{2}(0, T ; Y)$. One can pose the following questions: what information on the operator $A$ could be recovered from the observation $y(t)$ ? We mention works on the multidimensional inverse problems for the Schrödinger, heat and wave equations by one measurement, concerning this subject. Some of the results (for the Schrödinger equation) are given in $[9,10,16]$. To answer this question in the abstract setting, in [15] the authors derived the version of the BC-method equations under the condition that $A$ is self-adjoint and $Y=\mathbb{R}$. In the present paper, we address the same question without the assumption about selfajointness of $A$. The possibility of recovering the spectral data from the dynamical one is well-known for the dynamical system with a boundary control $[11,12]$. We extend these ideas to the case of the dual (observation) system.

The solvability of the BC-method equations for the spectral estimation problem critically depends on the properties of corresponding exponential family. The solvability of the BCmethod equations for system (1.2) depends on the controllability properties of the dual system. We point out the close relation between these two problems: they both leads to essentially the same equations (see section 4 for applications), and conditions for the solvability of these equations are the same (on the connections between the controllability of a dynamical systems and properties of exponential families see [5]).

In the second section, we outline the solution for the spectral estimation problem in infinite dimensional spaces (see [8] for details). In the third section, we derive the equations of the BC-method for problem (1.2), extending the results of [15] to the case of non self-adjoint operator. Also, we answer the question on the extension of the observation $y(t)=(\mathbb{O} a)(t)$. The last section is devoted to the applications to inverse problem by one measurement of the Schrödinger equation on the interval and to the problem of extending the inverse data for the first order hyperbolic system on the interval, see also [4,7-9].

\section{The spectral estimation problem in infinite dimensional spaces}

The problem is set up in the following way: given the signal (1.1), $S \in L_{2}(0,2 T)$, for $T>0$, to recover the coefficients $a_{k}(t), \lambda_{k}, k \in \mathbb{N}$. Below, we outline the procedure of recovering unknown parameters, for the details see [8].

We consider the dynamical systems in a complex Hilbert space $H$ :

$$
\begin{array}{ccc}
\dot{x}(t)=A x(t)+b f(t), & t \in(0, T), & x(0)=0 . \\
\dot{y}(t)=A^{*} y(t)+d g(t), & t \in(0, T), & y(0)=0,
\end{array}
$$

here $b, d \in H, f, g \in L_{2}(0, T)$, and we assume that the spectrum of the operator $A,\left\{\lambda_{k}\right\}_{k=1}^{\infty}$ is not simple. We denote the algebraic multiplicity of $\lambda_{k}$ by $L_{k}, k \in \mathbb{N}$, and also assume that the set of all root vectors $\left\{\phi_{k}^{i}\right\}, i=1, \ldots, L_{k}, k \in \mathbb{N}$, forms a Riesz basis in $H$. Here, the vectors from the chain $\left\{\phi_{k}^{i}\right\}_{i=1}^{L_{k}}, k \in \mathbb{N}$, satisfy the equations:

$$
\left(A-\lambda_{k}\right) \phi_{k}^{1}=0, \quad\left(A-\lambda_{k}\right) \phi_{k}^{i}=\phi_{k}^{i-1}, 2 \leqslant i \leqslant L_{k} .
$$


On some applications of the Boundary Control method to spectral estimation and...

The spectrum of $A^{*}$ is $\left\{\bar{\lambda}_{k}\right\}_{k=1}^{\infty}$ and the root vectors $\left\{\psi_{k}^{i}\right\}, i=1, \ldots, L_{k}, k \in \mathbb{N}$, also form a Riesz basis in $H$ and satisfy the equations:

$$
\left(A^{*}-\bar{\lambda}_{k}\right) \psi_{k}^{L_{k}}=0, \quad\left(A^{*}-\bar{\lambda}_{k}\right) \psi_{k}^{i}=\psi_{k}^{i+1}, 1 \leqslant i \leqslant L_{k}-1 .
$$

Moreover, the root vectors of $A$ and $A^{*}$ are normalized, in accordance with the following:

$$
\begin{array}{r}
\left\langle\phi_{k}^{i}, \psi_{l}^{j}\right\rangle=0, \text { if } k \neq l \text { or } i \neq j ; \\
\left\langle\phi_{k}^{i}, \psi_{k}^{i}\right\rangle=1, \quad i=1, \ldots, L_{k}, k \in \mathbb{N} .
\end{array}
$$

We consider $f$ and $g$ as the inputs of the systems (2.1) and (2.2) and define the outputs $z$ and $w$ by the formulas:

$$
z(t)=\langle x(t), d\rangle, \quad w(t)=\langle y(t), b\rangle .
$$

We assume that $b=\sum_{k=1}^{\infty} \sum_{i=1}^{L_{k}} b_{k}^{i} \phi_{k}^{i}, d=\sum_{k=1}^{\infty} \sum_{i=1}^{L_{k}} d_{k}^{i} \psi_{k}^{i}$. While searching for the solution to (2.1) in the form $x(t)=\sum_{k=1}^{\infty} \sum_{i=1}^{L_{k}} c_{k}^{i}(t) \phi_{k}^{i}$, we arrive at the following representation for the output:

$$
z(t)=\langle x(t), d\rangle=\sum_{k=1}^{\infty} \sum_{i=1}^{L_{k}} c_{k}^{i}(t) d_{k}^{i}=\int_{0}^{t} r(t-\tau) f(\tau) d \tau,
$$

where the response function $r(t)$ is defined as:

$$
r(t)=\sum_{k=1}^{\infty} e^{\lambda_{k} t}\left[a_{k}^{1}+a_{k}^{2} t+a_{k}^{3} \frac{t^{2}}{2}+\ldots+a_{k}^{L_{k}-1} \frac{t^{L_{k}-2}}{\left(L_{k}-2\right) !}+a_{k}^{L_{k}} \frac{t^{L_{k}-1}}{\left(L_{k}-1\right) !}\right],
$$

with $a_{k}^{j}$ being defined as:

$$
a_{k}^{j}=\sum_{i=j}^{L_{k}} b_{k}^{i} d_{k}^{i-j+1}, \quad j=1, \ldots, L_{k}, k \in \mathbb{N} .
$$

It is important to note that $r(t)$ has the form of the series in (1.1).

Analogously, looking for the solution of (2.2) in the form:

$$
y(t)=\sum_{k=1}^{\infty} \sum_{i=1}^{L_{k}} h_{k}^{i}(t) \psi_{k}^{i}
$$

we arrive at:

$$
w(t)=\langle y(t), b\rangle=\sum_{k=1}^{\infty} \sum_{i=1}^{L_{k}} h_{k}^{i}(t) b_{k}^{i}=\int_{0}^{t} \overline{r(t-\tau)} g(\tau) d \tau .
$$

We introduce the connecting operator $C^{T}: L_{2}(0, T) \mapsto L_{2}(0, T)$, defined through its bilinear form by the formula:

$$
\left\langle C^{T} f, g\right\rangle=\langle x(T), y(T)\rangle .
$$

In [8], the representation for $C^{T}$ was obtained:

Lemma 1. The connecting operator $C^{T}$ has a representation

$$
\left(C^{T} f\right)(t)=\int_{0}^{T} r(2 T-t-\tau) f(\tau) d \tau
$$


We assume that the systems (2.1), (2.2) are spectrally controllable in time $T$. This means that for any $i \in\left\{1, \ldots, L_{k}\right\}$, and any $k \in \mathbb{N}$, there exist $f_{k}^{i}, g_{k}^{i} \in H_{0}^{1}(0, T)$, such that $x^{f_{k}^{i}}(T)=\phi_{k}^{i}, y^{g_{k}^{i}}(T)=\psi_{k}^{i}$. Using ideas of the BC method [13], we are able to extract the spectral data, $\left\{\lambda_{k}, a_{k}^{j}\right\}, j=1, \ldots, L_{k}, k \in \mathbb{N}$, from the dynamical one, $r(t), t \in(0,2 T)$, (see $[4,8]$ for more details):

Proposition 1. The set $\lambda_{k}, f_{k}^{i}, i=1, \ldots, L_{k}, k \in \mathbb{N}$, are eigenvalues and root vectors of the following generalized eigenvalue problem in $L_{2}(0, T)$ :

$$
\int_{0}^{T}\left(r^{\prime}(2 T-t-\tau)-\lambda r(2 T-t-\tau)\right) f(\tau) d \tau=0 .
$$

The set $\bar{\lambda}_{k}, g_{k}^{i}, k=1, \ldots \infty, i=1, \ldots, L_{k}$ are eigenvalues and root vectors of the generalized eigenvalue problem in $L_{2}(0, T)$ :

$$
\int_{0}^{T}\left(\overline{r^{\prime}(2 T-t-\tau)}-\lambda \overline{r(2 T-t-\tau)}\right) g(\tau) d \tau=0 .
$$

Now, we describe the algorithm of recovering $a_{k}^{1}, \ldots a_{k}^{L_{k}}, k \in \mathbb{N}$ (see the representation (2.3)). We normalize the solutions to (2.5), (2.6) by the rule:

$$
\left\langle C^{T} \widetilde{f}_{k}^{i}, \widetilde{g}_{k}^{i}\right\rangle=1
$$

and define:

$$
\begin{aligned}
& \widetilde{b}_{k}^{i}=\left\langle y^{\widetilde{g}_{k}^{i}}(T), b\right\rangle=\int_{0}^{T} \bar{r}(T-\tau) \widetilde{g}_{k}^{i}(\tau) d \tau, \\
& \widetilde{d}_{k}^{i}=\left\langle x^{\widetilde{f}_{k}^{i}}(T), d\right\rangle=\int_{0}^{T} r(T-\tau) \widetilde{f}_{k}^{i}(\tau) d \tau .
\end{aligned}
$$

Then (see (2.4))

$$
a_{k}^{1}=\sum_{i=1}^{L_{k}} \widetilde{b}_{k}^{i} \widetilde{d}_{k}^{i}
$$

We denote by $\partial$ and $I$ the differentiation operator and the identity operator in $L_{2}(0, T)$. We normalize the solutions to (2.5), (2.6) (for $i>l$ ) by the following rule:

$$
\left\langle\left[C^{T}\left(\partial-\lambda_{k} I\right)\right]^{l} \widehat{f}_{k}^{i}, \widehat{g}_{k}^{i-l}\right\rangle=1,
$$

we define $\widehat{b}_{k}^{i}, \widehat{d}_{k}^{i}$ by (2.8), (2.9) and evaluate:

$$
a_{k}^{l}=\sum_{i=l}^{L_{k}} \widehat{b}_{k}^{i} \widehat{d}_{k}^{-l+1}, \quad l=2, \ldots, L_{k} .
$$

We conclude this section with the algorithm for solving the spectral estimation problem: suppose that we are given with the function $r \in L_{2}(0,2 T)$ of the form (2.3) and the family $\bigcup_{k=1}^{\infty}\left\{e^{\lambda_{k} t}, \ldots, t^{L_{k}-1} e^{\lambda_{k} t}\right\}$ is minimal in $L_{2}(0, T)$. Then, to recover $\lambda_{k}, L_{k}$ and coefficients of polynomials, one should utilize the following: 
On some applications of the Boundary Control method to spectral estimation and...

\section{Algorithm}

a) solve generalized eigenvalue problems (2.5), (2.6) to find $\lambda_{k}, L_{k}$ and non-normalized controls.

b) Normalize $\widetilde{f}_{k}^{i}, \widetilde{g}_{k}^{i}$ by (2.7), define $\widetilde{b}_{k}^{i}, \widetilde{d}_{k}^{i}$ by (2.8), (2.9) to recover $a_{k}^{1}$ by (2.10).

c) Normalize $\widehat{f}_{k}^{i}, \widehat{g}_{k}^{i-l}$ by (2.11), define $\widehat{b}_{k}^{i}, \widehat{d}_{k}^{i}$ by (2.8), (2.9) to recover $a_{k}^{l}$ by (2.12), $l=2, \ldots, L_{k}-1$.

\section{Equations of the $B C$ method}

Let us denote by $A^{*}$ the operator adjoint to $A$ and $B:=O^{*}, B: Y \mapsto H$. Along with system (1.2), we consider the following dynamical control system:

$$
\left\{\begin{array}{l}
v_{t}+A^{*} v=B f, \quad t<T \\
v(T)=0
\end{array}\right.
$$

and denote its solution by $v^{f}$. The reason we consider the system (3.1) reverse in time is that it is adjoint to $(1.2)$ (see $[5,15])$.

For every $0 \leqslant s<T$, we introduce the control operator by $W^{s} f:=v^{f}(s)$. It is easy to verify that $-W^{0}$ is adjoint to $\mathbb{O}^{T}$. Indeed, taking $f \in L_{2}(0, T ; Y), a \in H$ we show [15] that:

$$
\int_{0}^{T}(f, \mathbb{O} a)_{Y}=-\left(W^{0} f, a\right)_{H}
$$

here $\mathbb{O} a=\left(O u^{a}\right)(t)$. Due to the arbitrariness of $f$ and $a$, the last equality is equivalent to $\left(\mathbb{O}^{T}\right)^{*}=-W^{0}$.

We assume that the operator $A$ satisfies the following assumptions:

Assumption 1. a) The spectrum of the operator $A,\left\{\lambda_{k}\right\}_{k=1}^{\infty}$ consists of the eigenvalues $\lambda_{k}$ with algebraic multiplicity $L_{k}, k \in \mathbb{N}$, and the set of all root vectors $\left\{\phi_{k}^{i}\right\}, i=1, \ldots, L_{k}$, $k \in \mathbb{N}$, form a Riesz basis in $H$. Here, the vectors from the chain $\left\{\phi_{k}^{i}\right\}_{i=1}^{L_{k}}, k \in \mathbb{N}$, satisfy the equations

$$
\left(A-\lambda_{k}\right) \phi_{k}^{1}=0, \quad\left(A-\lambda_{k}\right) \phi_{k}^{i}=\phi_{k}^{i-1}, 2 \leqslant i \leqslant L_{k} .
$$

The root vectors of $A^{*},\left\{\psi_{k}^{i}\right\}, i=1, \ldots, L_{k}, k \in \mathbb{N}$, form a Riesz basis in $H$ and satisfy:

$$
\left(A^{*}-\bar{\lambda}_{k}\right) \psi_{k}^{L_{k}}=0, \quad\left(A^{*}-\bar{\lambda}_{k}\right) \psi_{k}^{i}=\psi_{k}^{i+1}, 1 \leqslant i \leqslant L_{k}-1 .
$$

b) The system (3.1) is spectrally controllable in time $T$ : i.e. there exists the controls $f_{k}^{i} \in H_{0}^{1}(0, T ; Y)$ such that $W^{0} f_{k}^{i}=\psi_{k}^{i}$, for $i=1, \ldots, L_{k}, k \in \mathbb{N}$.

We say that the vector $a$ is generic if its Fourier representation in the basis $\left\{\phi_{k}^{i}\right\}_{k=1}^{\infty}$, $a=\sum_{k=1}^{\infty} \sum_{i=1}^{L_{k}} a_{k}^{i} \phi_{k}^{i}$, is such that $a_{k}^{i} \neq 0$ for all $k, i$. We assume that the controls from the Assumption 1 are extended by zero outside the interval $(0, T)$. Now, we are ready to formulate.

Theorem 1. If A satisfies Assumption $1, Y=\mathbb{R}$, and source a is generic, then the spectrum of $A$ and controls $f_{k}^{i}$ are the spectrum and the root vectors of the following generalized spectral problem:

$$
\int_{0}^{2 T}\left((\dot{O a})(t)-\lambda_{k}(O a)(t), f_{k}(t-T+\tau)\right)_{Y} d t=0, \quad 0<\tau<T .
$$

Here, by dot, we denote the differentiation with respect to $t$. 
Proof. We denote by $\left\{\tilde{f}_{k}^{i}\right\}$ the set of controls which satisfy $W^{0} \widetilde{f}_{k}^{i}=\psi_{k}^{i}$. By $\left\{f_{k}^{i}\right\}$ we denote the set of shifted controls: $f_{k}^{i}(t)=\widetilde{f}_{k}^{i}(t-T)$. Thus, the control $f_{k}^{i}$ acts on the time interval $(T, 2 T)$. Let us fix some $i \in 1, \ldots, L_{k}, k \in \mathbb{N}, \tau \in(0, T)$ and consider $W^{0}\left(\dot{f}_{k}^{i}(\cdot+\tau)\right)$ :

$$
W^{0}\left(\dot{f}_{k}^{i}(\cdot+\tau)\right)=v^{\dot{f}_{k}^{i}(\cdot+\tau)}(0)=v_{t}^{f_{k}^{i}(\cdot+\tau)}(0)=\left(B f_{k}^{i}(\cdot+\tau)\right)(0)-A^{*} v_{k}^{f_{k}^{i}(\cdot+\tau)}(0) .
$$

Since $f_{k}^{i} \in H_{0}^{1}(T, 2 T, Y),\left(B f_{k}^{i}(\cdot+\tau)\right)(0)=0$. The second term on the right side of (3.4) could be evaluated using the following reasons. The function $v^{f_{k}^{i}}$ solves:

$$
\begin{gathered}
v_{t}^{f_{k}^{i}(\cdot+\tau)}+A^{*} v_{k}^{f_{k}^{i}(\cdot+\tau)}=0, \quad 0 \leqslant t \leqslant T-\tau, \\
v_{k}^{f_{k}^{i}(\cdot+\tau)}(T-\tau)=\psi_{k}^{i} .
\end{gathered}
$$

We are looking for a solution in the form $v^{f_{k}^{i}(\cdot+\tau)}(t)=\sum_{j=1}^{L_{k}} c_{k}^{j}(t) \psi_{k}^{j}$, then $c_{k}^{j}$ satisfies boundary conditions $c_{k}^{j}(0)=\delta_{i j}$ and equation:

$$
\begin{aligned}
& \frac{d}{d t} c_{k}^{1}+\bar{\lambda}_{k} c_{k}^{1}=0, \\
& \frac{d}{d t} c_{k}^{j}+\bar{\lambda}_{k} c_{k}^{j}+c_{k}^{j-1}=0, \quad j=2, \ldots, L_{k} .
\end{aligned}
$$

Solving this system, we obtain the following expansion:

$$
v^{f_{k}^{i}(\cdot+\tau)}(t)=\sum_{j=i}^{L_{k}} \frac{(T-\tau-t)^{j-i}}{(j-i) !} e^{\bar{\lambda}_{k}(T-\tau-t)} \psi_{k}^{j} .
$$

Evaluating $A^{*} v_{k}^{f_{k}^{i}(\cdot+\tau)}(0)$, making use of (3.5) and properties of the root vectors, we arrive at:

$$
\begin{aligned}
& A^{*} v^{f_{k}^{L_{k}}(\cdot+\tau)}(0)=\bar{\lambda}_{k} v^{f_{k}^{L_{k}}(\cdot+\tau)}(0), \\
& A^{*} v^{f_{k}^{i}(\cdot+\tau)}(0)=\bar{\lambda}_{k} v^{f_{k}^{i}(\cdot+\tau)}(0)+v^{f_{k}^{i+1}(\cdot+\tau)}(0), i<L_{k} .
\end{aligned}
$$

Then, continuing (3.4), we obtain:

$$
\begin{gathered}
W^{0}\left(\dot{f}_{k}^{L_{k}}(\cdot+\tau)\right)=-A^{*} v^{f_{k}^{L_{k}}(\cdot+\tau)}(0)=-\bar{\lambda}_{k} W^{0} f_{k}^{L_{k}}, \\
W^{0}\left(\dot{f}_{k}^{i}(\cdot+\tau)\right)=-\bar{\lambda}_{k} W^{0} f_{k}^{i}-\bar{\lambda}_{k} W^{0} f_{k}^{i+1}, i<L_{k} .
\end{gathered}
$$
we get:

Integrating by parts and taking into account that $f_{k}^{i}(0)=f_{k}^{i}(T)=0$ for $i=1, \ldots, L_{k}$,

$$
\begin{gathered}
\int_{0}^{2 T}\left((O a)(t), \dot{f}_{k}^{i}(t+\tau)\right)_{Y} d t=-\int_{0}^{2 T}\left((\dot{O} a)(t), f_{k}^{i}(t+\tau)\right)_{Y} d t \\
+\left.\left((\dot{O a})(t+\tau), f_{k}^{i}(t)\right)_{Y}\right|_{t=0} ^{t=2 T}=-\int_{0}^{2 T}\left((\dot{O} a)(t), f_{k}^{i}(t+\tau)\right)_{Y} d t
\end{gathered}
$$


Conversely, using the duality between $W^{0}$ and $\mathbb{O}^{T}$ and (3.6), (3.7), we have for $i=L_{k}$ :

$$
\begin{array}{r}
\int_{0}^{2 T}\left((O a)(t), \dot{f}_{k}^{L_{k}}(t+\tau)\right)_{Y} d t=-\left(a, W^{0} \dot{f}_{k}^{L_{k}}(\cdot+\tau)\right)_{H}=\left(a, \bar{\lambda}_{k} W^{0} f_{k}^{L_{k}}(\cdot+\tau)\right)_{H}= \\
\left(\lambda_{k} a, W^{0} f_{k}^{L_{k}}(\cdot+\tau)\right)_{H}=-\int_{0}^{2 T}\left(\lambda_{k}(O a)(t), f_{k}^{L_{k}}(t+\tau)\right)_{Y} d t
\end{array}
$$

and for $i<L_{k}$ :

$$
\begin{array}{r}
\int_{0}^{2 T}\left((O a)(t), \dot{f}_{k}^{i}(t+\tau)\right)_{Y} d t=\left(a, \bar{\lambda}_{k} W^{0} f_{k}^{i}(\cdot+\tau)+W^{0} f_{k}^{i+1}(\cdot+\tau)\right)_{H}= \\
-\lambda_{k} \int_{0}^{2 T}\left((O a)(t), f_{k}^{i}(t+\tau)\right)_{Y} d t-\int_{0}^{2 T}\left((O a)(t), f_{k}^{i+1}(t+\tau)\right)_{Y} d t
\end{array}
$$

In what follows, we assume that elements with index $i=L_{k}+1$ or $i=0$ are zero. Combining (3.8) and (3.9), (3.10), we see that the pair $\lambda_{k}, f_{k}$ satisfies on $0<\tau<T, i=$ $1, \ldots, L_{k}$ :

$$
\int_{0}^{2 T}\left((\dot{O a})(t)-\lambda_{k}(O a)(t), f_{k}^{i}(t+\tau)\right)_{Y} d t=\int_{0}^{2 T}\left((O a)(t), f_{k}^{i+1}(t+\tau)\right)_{Y} d t .
$$

Now we prove the converse; solving the generalized eigenvalue problem:

$$
\int_{0}^{2 T}((\dot{O} a)(t)-\lambda(O a)(t), f(t+\tau))_{Y} d t=0
$$

yields $\left\{\lambda_{k}\right\}_{k=1}^{\infty}$ eigenvalues of $A$ and controls $\left\{f_{k}^{i}\right\}, i=1, \ldots, L_{k}, k \in \mathbb{N}$.

Let the functions $\left\{f_{1}, \ldots, f_{L}\right\}$ satisfying (3.11) constitute the chain for (3.12) for some $\lambda$. Then, as it follows from the proof that for $\tau \in(0, T)$ :

$$
\left(a, W^{0} \dot{f}_{i}(t+\tau)\right)_{H}+\lambda\left(a, W^{0} f_{i}(t+\tau)\right)_{H}=-\left(a, W^{0} f_{i+1}(t+\tau)\right)_{H},
$$

which is equivalent to

$$
-\left(a, A^{*} v^{f_{i}(t+\tau)}(0)\right)_{H}+\lambda\left(a, v^{f_{i}(t+\tau)}(0)\right)_{H}=-\left(a, v^{f_{i+1}(t+\tau)}(0)\right)_{H}, \tau \in(0, T) .
$$

First, we consider case $i=L$. We rewrite the last equality (using the notation $f=f_{L}$ ) as:

$$
\left(a, A^{*} v^{f(t+\tau)}(0)-\bar{\lambda} v^{f(t+\tau)}(0)\right)_{H}=0, \quad \tau \in(0, T) .
$$

We assume that $v^{f(t+\tau)}(T-\tau)=\sum_{\substack{k \in \mathbb{N} \\ i=1, \ldots, L_{k}}} c_{k}^{i} \psi_{k}^{i}$. Then, developing $v^{f}$ in the Fourier series as we did in (3.5), we arrive at:

$$
v^{f(t+\tau)}(0)=\sum_{\substack{k \in \mathbb{N} \\ i=1, \ldots, L_{k}}} c_{k}^{i} \sum_{j=1}^{L_{k}} \frac{(T-\tau)^{j-i}}{(j-i) !} e^{\bar{\lambda}_{k}(T-\tau)} \psi_{k}^{j}
$$


Applying operator $A^{*}$ and using the property $A^{*} \psi_{k}^{j}=\bar{\lambda}_{k} \psi_{k}^{j}+\psi_{k}^{j+1}$, we obtain:

$$
A^{*} v^{f(t+\tau)}(0)=\sum_{\substack{k \in \mathbb{N} \\ i=1, \ldots, L_{k}}} c_{k}^{i} \sum_{j=1}^{L_{k}} \frac{(T-\tau)^{j-i}}{(j-i) !} e^{\bar{\lambda}_{k}(T-\tau)}\left(\bar{\lambda}_{k} \psi_{k}^{j}+\psi_{k}^{j+1}\right) .
$$

Introducing the notation:

$$
g(\tau):=A^{*} v^{f(t+\tau)}(0)-\bar{\lambda} v^{f(t+\tau)}(0)=\sum_{\substack{k \in \mathbb{N} \\ i=1, \ldots, L_{k}}} g_{k}^{i}(\tau) \psi_{k}^{i}
$$

relation (3.14) yields:

$$
0=(a, g)_{H}=\sum_{\substack{k \in \mathbb{N} \\ i=1, \ldots, L_{k}}} a_{k}^{i} g_{k}^{i}(\tau), \quad \tau \in(0, T) .
$$

The functions $g_{k}^{i}(\tau)$ are combination of products of $e^{\bar{\lambda}_{k}(T-\tau)}$ and polynomials $\frac{(T-\tau)^{\alpha}}{\alpha !}$. Then, we can rewrite (3.18) as follows:

$$
0=\sum_{\substack{k \in \mathbb{N} \\ i=1, \ldots, L_{k}}} b_{k}^{i} \frac{(T-\tau)^{i-1}}{(i-1) !} e^{\bar{\lambda}_{k}(T-\tau)}, \quad \tau \in(0, T) .
$$

If $Y=\mathbb{R}$, the controllability of the dynamical system (3.1) imply [5] the minimality of the family $\bigcup_{k=1}^{\infty}\left\{e^{\bar{\lambda}_{k} t}, t e^{\bar{\lambda}_{k} t} \ldots, t^{L_{k}-1} e^{\bar{\lambda}_{k} t}\right\}$ in $L_{2}(0, T)$ in $L_{2}(0, T)$, so we have $b_{k}^{i}=0$ for all $k, i$. However, as follows from (3.15), (3.16):

$$
b_{k}^{L_{k}}=c_{k}^{1} \bar{\lambda}_{k} a_{k}^{1}-\bar{\lambda} c_{k}^{1} a_{k}^{1}=0 .
$$

Then, since $a$ is generic, either $\lambda=\lambda_{k}$ or $c_{k}^{1}=0$.

Let $\lambda \neq \lambda_{k}$, so $c_{k}^{1}=0$. Then, for $b_{k}^{L_{k}-1}$, we have:

$$
b_{k}^{L_{k}-1}=c_{k}^{2} \bar{\lambda}_{k} a_{k}^{2}-\bar{\lambda} c_{k}^{2} a_{k}^{2}=0,
$$

from which the equality $c_{k}^{2}=0$ follows. Repeating this procedure for $b_{k}^{L_{k}-i}, i \geqslant 2$, we obtain:

$$
\text { If } \lambda \neq \lambda_{k} \text {, then } c_{k}^{i}=0, i=1, \ldots, L_{k} \text {. }
$$

We consider the second option; let $\lambda=\lambda_{k}$. Then, from (3.15) and (3.16):

$$
b_{k}^{L_{k}-1}=c_{k}^{1}=0, \quad b_{k}^{L_{k}-2}=c_{k}^{2} a_{k}^{3}=0, \ldots, b_{k}^{1}=c_{k}^{L_{k}-1} a_{k}^{L_{k}}=0 .
$$

So, we arrive at the following:

$$
\text { If } \lambda=\lambda_{k} \text {, then } c_{k}^{i}=0, i=1, \ldots, L_{k}-1 \text {, and } c_{k}^{L_{k}} \text { could be arbitrary. }
$$

Finally (3.20), (3.21) imply that $\lambda=\lambda_{k^{\prime}}$ and $f=c_{k^{\prime}} f_{k^{\prime}}^{L_{k^{\prime}}}, c_{k^{\prime}} \neq 0$, for some $k^{\prime}$.

Thus, on the first step we already obtained that $\lambda=\lambda_{k^{\prime}}$ for some $k^{\prime}$ and $f_{L}=c_{k^{\prime}} f_{k^{\prime}}^{L_{k^{\prime}}}$. The second vector $f$ in the Jordan chain satisfies

$$
\int_{0}^{2 T}\left((\dot{O a})(t)-\lambda_{k^{\prime}}(O a)(t), f(t+\tau)\right)_{Y} d t=\int_{0}^{2 T}\left((O a)(t), c_{k^{\prime}} f_{k^{\prime}}^{L_{k^{\prime}}}(t+\tau)\right)_{Y} d t .
$$

We rewrite (3.13) in our case:

$$
-\left(a, A^{*} v^{f(t+\tau)}(0)\right)_{H}+\lambda_{k^{\prime}}\left(a, v^{f(t+\tau)}(0)\right)_{H}=-\left(a, c_{k^{\prime}} v^{f_{k^{\prime}}^{L_{k^{\prime}}}(t+\tau)}(0)\right)_{H}, \tau \in(0, T) .
$$


In this case, $g$, introduced in (3.17), has the form:

$$
g(\tau)=\sum_{\substack{k \in \mathbb{N} \\ i=1, \ldots, L_{k}}} c_{k}^{i} \sum_{j=1}^{L_{k}} \frac{(T-\tau)^{j-i}}{(j-i) !} e^{\bar{\lambda}_{k}(T-\tau)}\left(\left(\bar{\lambda}_{k}-\bar{\lambda}_{k^{\prime}}\right) \psi_{k}^{j}+\psi_{k}^{j+1}\right),
$$

and rewrite (3.22) as:

$$
(a, g)_{H}=\left(a, v^{f_{k^{\prime}}^{L^{\prime}}(\cdot+\tau)}\right)_{H}=c_{k^{\prime}} a_{k^{\prime}}^{L_{k^{\prime}}} e^{\bar{\lambda}_{k^{\prime}}(T-\tau)}
$$

Using the same notations in (3.18) and (3.19), we transcribe the equalities for coefficients $b_{k}^{i}$ for (3.23) to get:

$$
b_{k^{\prime}}^{1}=c_{k^{\prime}} a_{k^{\prime}}^{L_{k^{\prime}}}, \quad b_{k}^{i}=0, \quad k \neq k^{\prime}
$$

In the case $k \neq k^{\prime}$, we repeat the arguments used above and find that:

$$
c_{k}^{i}=0, \quad i=1, \ldots, L_{k} .
$$

When $k=k^{\prime}$, we have:

$$
\begin{array}{r}
b_{k^{\prime}}^{L_{k^{\prime}}}=0, \quad b_{k^{\prime}}^{L_{k^{\prime}}-1}=c_{k^{\prime}}^{1} a_{k^{\prime}}^{L_{k^{\prime}}}=0, \quad b_{k^{\prime}}^{L_{k^{\prime}}-2}=c_{k^{\prime}}^{2} a_{k^{\prime}}^{L_{k^{\prime}}}=0, \\
b_{k^{\prime}}^{2}=c_{k^{\prime}}^{L_{k^{\prime}}-2} a_{k^{\prime}}^{L_{k^{\prime}}}=0, \quad b_{k^{\prime}}^{1}=c_{k^{\prime}}^{L_{k^{\prime}}-1} a_{k^{\prime}}^{L_{k^{\prime}}}=c_{k^{\prime}} a_{k^{\prime}}^{L_{k^{\prime}}} .
\end{array}
$$

So, we find:

$$
c_{k^{\prime}}^{i}=0, \quad i<L_{k^{\prime}}-1, \quad c_{k^{\prime}}^{L_{k^{\prime}}-1}=c_{k^{\prime}}, \quad c_{k^{\prime}}^{L_{k^{\prime}}} \text { is arbitrary. }
$$

So, finally we arrive at for some $c_{L-1}$ :

$$
f=f_{L-1}=c_{k^{\prime}} f_{k^{\prime}}^{L_{k^{\prime}}-1}+c_{L-1} f_{k^{\prime}}^{L_{k^{\prime}}}
$$

Arguing in the same fashion, we obtain that:

$$
f_{i}=c_{k^{\prime}} f_{k^{\prime}}^{L_{k^{\prime}}-i}+c_{i} f_{k^{\prime}}^{L_{k^{\prime}}}, \quad 1 \leqslant i<L_{k^{\prime}}-1 .
$$

So, we have shown that the elements of the Jordan chain for (3.3) which correspond to eigenvalue $\lambda_{k^{\prime}}$ are the linear combination of corresponding controls and eigenvector (i.e. the control that generate the eigenvector of $A^{*}$ ).

Remark 1. The solution to (3.3) yields $\left\{\lambda_{k}\right\}_{k=1}^{\infty}$ eigenvalues of $A$ and (non-normalized) root vectors $\left\{\widehat{f}_{k}^{i}\right\}, \widehat{f}_{k}^{i}=c_{k} f_{k}^{i}+c_{k}^{i} f_{k}^{L_{k}} k \in \mathbb{N}, i=1, \ldots, L_{k}, c_{k}^{L_{k}}=0$.

For the dynamical system (1.2), under the conditions on $A, Y$, formulated in Theorem 1 , there is the possibility to extend the observation $y(t)=\left(O u^{a}\right)(t)$ defined for $t \in(0,2 T)$ to $t \in \mathbb{R}_{+}$. To this end, we show that for an observation having the form:

$$
\mathbb{O} a=\sum_{k \in \mathbb{N}} e^{\lambda_{k} t} \sum_{j=1}^{L_{k}} \frac{b_{k}^{j} t^{L_{k}-j}}{\left(L_{k}-j\right) !},
$$

we can recover the coefficients $b_{k}^{j}$.

Take $i \in\left\{1, \ldots, L_{k}\right\}$ and search for the solution to (1.2) with $a=\phi_{k}^{i}$ in the form $u=\sum_{l=1}^{L_{k}} c_{l}(t) \phi_{k}^{l}$, we arrive at the system (here $c_{L_{k}+1}=0$ ):

$$
\begin{array}{r}
\frac{d}{d t} c_{l}(t)-\lambda_{k} c_{l}(t)=c_{l+1}(t), \quad l=1, \ldots, L_{k}, \\
c_{l}(0)=\delta_{l i} .
\end{array}
$$


whose solution is:

$$
\begin{array}{r}
c_{l}(t)=\frac{t^{i-l}}{(i-l) !} e^{\lambda_{k} t}, \quad l \leqslant i, \\
c_{l}(t)=0, \quad l>i .
\end{array}
$$

Thus,

$$
u^{\phi_{k}^{i}}=\sum_{l=1}^{i} \frac{t^{i-l}}{(i-l) !} e^{\lambda_{k} t} \phi_{k}^{l} .
$$

For the initial state $a=\sum_{k \in \mathbb{N}} \sum_{i=1}^{L_{k}} a_{k}^{i} \phi_{k}^{i}$, we obtain:

$$
u^{a}=\sum_{k \in \mathbb{N}} e^{\lambda_{k} t} \sum_{j=1}^{L_{k}} \frac{t^{L_{k}-j}}{\left(L_{k}-j\right) !} \sum_{l=1}^{j} a_{k}^{L_{k}-j+l} \phi_{k}^{l} .
$$

So, for observation $(\mathbb{O} a)(t)=\left(O u^{a}\right)(t)$, we derive the representation (3.24) with coefficients $b_{k}^{j}$, defined by:

$$
b_{k}^{j}:=\sum_{l=1}^{j} a_{k}^{L_{k}-j+l} O \phi_{k}^{l}, \quad k \in \mathbb{N}, j=1, \ldots, L_{k} .
$$

Making use of Theorem 1 (see also Remark 1), we have:

$$
W^{0} \widehat{f}_{k}^{i}=c_{k} \psi_{k}^{i}+c_{k}^{i} \psi_{k}^{L_{k}}, \quad k \in \mathbb{N}, i=1, \ldots, L_{k}, c_{k}^{L_{k}}=0 .
$$

Counting (3.2), we write:

$$
\left(W^{0} \widehat{f}_{k}^{\imath}, a\right)_{H}=-\int_{0}^{T} O u^{a} \widehat{f}_{k}^{\imath} d t .
$$

We plug $a=\phi_{k}^{i}$ into the last equality and use (3.27) to get:

$$
c_{k}=\left(c_{k} \psi_{k}^{i}+c_{k}^{i} \psi_{k}^{L_{k}}, \phi_{k}^{i}\right)_{H}=-\int_{0}^{T} O u^{\phi_{k}^{i}} \widehat{f_{k}^{i}} d t .
$$

We evaluate the right side of (3.28) for all $i$. For $i=1$ we get (see (3.25)):

$$
c_{k}=-O \phi_{k}^{1} \int_{0}^{T} e^{\lambda_{k} t} \widehat{f}_{k}^{1} d t .
$$

Or equivalently:

$$
\frac{c_{k}}{O \phi_{k}^{1}}=-\int_{0}^{T} e^{\lambda_{k} t} \widehat{f}_{k}^{1} d t
$$

Evaluating (3.28) for $i=2$, counting (3.25), we obtain:

$$
c_{k}=-O \phi_{k}^{2} \int_{0}^{T} e^{\lambda_{k} t} \widehat{f}_{k}^{2} d t-O \phi_{k}^{1} \int_{0}^{T} t e^{\lambda_{k} t} \widehat{f}_{k}^{2} d t .
$$


We divide this equality by $c_{k}$ and plug (3.29) in to find:

$$
\frac{c_{k}}{O \phi_{k}^{2}}=-\frac{\int_{0}^{T} e^{\lambda_{k} t} \widehat{f}_{k}^{1} d t \int_{0}^{T} e^{\lambda_{k} t} \widehat{f}_{k}^{2} d t}{\int_{0}^{T} e^{\lambda_{k} t} \widehat{f}_{k}^{1} d t-\int_{0}^{T} t e^{\lambda_{k} t} \widehat{f}_{k}^{2} d t}
$$

Suppose we already found $\frac{c_{k}}{O \phi_{k}^{l}}$ for $l=1, \ldots, i-1$. To find this quantity for $l=i$, we evaluate (3.28), plugging the expression for $u^{\phi_{k}^{i}}(3.25)$ :

$$
c_{k}=-\sum_{l=1}^{i} O \phi_{k}^{l} \int_{0}^{T} \frac{t^{i-l}}{(i-l) !} e^{\lambda_{k} t} \widehat{f}_{k}^{i} d t .
$$

We divide last equality by $c_{k}$ to find:

$$
\frac{c_{k}}{O \phi_{k}^{i}}=-\frac{\int_{0}^{T} e^{\lambda_{k} t} \widehat{f}_{k}^{i} d t}{1+\sum_{l=1}^{i-1} \int_{0}^{T} \frac{t^{i-l}}{(i-l) !} e^{\lambda_{k} t} \widehat{f}_{k}^{i} d t\left(\frac{c_{k}}{O \phi_{k}^{l}}\right)^{-1}} .
$$

Observe that in the right side of (3.31) in view of (3.30), we know all terms.

To evaluate $a_{k}^{i}$, we use, see (3.27):

$$
a_{k}^{i}=\left(a, \psi_{k}^{i}\right)_{H}=\left(a, W^{0} \widehat{f}_{k}^{i}-c_{k}^{i} \psi_{k}^{L_{k}}\right)_{H} \frac{1}{c_{k}}=-\int_{0}^{T} O u^{a} \widehat{f_{k}^{i}} d t \frac{1}{c_{k}}-a_{k}^{L_{k}} \frac{c_{k}^{i}}{c_{k}}
$$

We multiply (3.27) by $\phi_{k}^{L_{k}}$ and obtain for $i<L_{k}$ :

$$
\begin{array}{r}
c_{k}^{i}=\left(W^{0} f_{k}^{i}, \phi_{k}^{L_{k}}\right)_{H}=-\int_{0}^{T} f_{k}^{i}(t)\left(O u^{\phi_{k}^{L_{k}}}\right)(t) d t \\
=-\sum_{l=1}^{L_{k}} O \phi_{k}^{l} \int_{0}^{T} \frac{t^{L_{k}-l}}{\left(L_{k}-l\right) !} e^{\lambda_{k} t} f_{k}^{i}(t) d t .
\end{array}
$$

Dividing the last equality by $c_{k}$, we obtain:

$$
\frac{c_{k}^{i}}{c_{k}}=-\sum_{l=1}^{L_{k}}\left(\frac{c_{k}}{O \phi_{k}^{l}}\right)^{-1} \int_{0}^{T} \frac{t^{L_{k}-l}}{\left(L_{k}-l\right) !} e^{\lambda_{k} t} f_{k}^{i}(t) d t, \quad i<L_{k} .
$$

Notice that in view of (3.31), we know all terms in the right hand side in (3.33). Now, we multiply (3.32) by $c_{k}$ :

$$
a_{k}^{i} c_{k}=-\int_{0}^{T} O u^{a} \widehat{f}_{k}^{i} d t-a_{k}^{L_{k}} c_{k} \frac{c_{k}^{i}}{c_{k}} .
$$

Since $c_{k}^{L_{k}}=0$, we have for $i=L_{k}$ :

$$
a_{k}^{L_{k}} c_{k}=-\int_{0}^{T} \widehat{f}_{k}^{L_{k}}(t)\left(O u^{a}\right)(t) d t
$$


and finally:

$$
a_{k}^{i} c_{k}=-\int_{0}^{T} \widehat{f}_{k}^{i}(t)\left(O u^{a}\right)(t) d t+\int_{0}^{T} \widehat{f}_{k}^{L_{k}}(t)\left(O u^{a}\right)(t) d t \frac{c_{k}^{i}}{c_{k}} .
$$

In view of (3.33), we know all terms on the right side of (3.34).

Now, we rewrite formula for $b_{k}^{j}(3.26)$ :

$$
b_{k}^{j}:=\sum_{l=1}^{j}\left\{a_{k}^{L_{k}-j+l} c_{k}\right\}\left(\frac{O \phi_{k}^{l}}{c_{k}}\right) \quad k \in \mathbb{N}, j=1, \ldots, L_{k},
$$

and observe that the first term in each summand is given by (3.34), while the second term by (3.31). So, we know right hand side in (3.35).

After we recovered all $b_{k}^{j}$ by (3.35), we can extend the observation $(\mathbb{O} a)(t)$ by formula (3.24) for $t>2 T$.

\section{Application to inverse problems}

Here, we provide two applications of the above-developed theory to inverse problems. Other applications of the $\mathrm{BC}$ approach to the spectral estimation problem can be found in $[1,2,4,7-9,15]$.

\subsection{Reconstructing the potential for the 1D Schrödinger equation from boundary measurements}

Let the real potential $q \in L^{1}(0,1)$ and $a \in H_{0}^{1}(0,1)$ be fixed, we consider the boundary value problem:

$$
\begin{cases}i u_{t}(x, t)-u_{x x}(x, t)+q(x) u(x, t)=0 & t>0, \quad 0<x<1 \\ u(0, t)=u(1, t)=0 & t>0, \\ u(x, 0)=a(x) & 0<x<1 .\end{cases}
$$

Assuming that the initial datum $a$ is generic (but unknown), the inverse problem we are interested in is to determine the potential $q$ from the trace of the derivative of the solution $u$ to (4.1) on the boundary:

$$
\left\{r_{0}(t), r_{1}(t)\right\}:=\left\{u_{x}(0, t), u_{x}(1, t)\right\}, \quad t \in(0,2 T),
$$

It is well known that the self-adjoint operator $A$ defined on $L^{2}(0,1)$ by:

$$
A \phi=-\phi^{\prime \prime}+q \phi, \quad D(A):=H^{2}(0,1) \cap H_{0}^{1}(0,1),
$$

admits a family of eigenfunctions $\left\{\phi_{k}\right\}_{k=1}^{\infty}$ forming a orthonormal basis in $L^{2}(0,1)$, and associated sequence of eigenvalues $\lambda_{k} \rightarrow+\infty$. Using the Fourier method, we can represent the solution of (4.1) in the form:

$$
u(x, t)=\sum_{k=1}^{\infty} a_{k} e^{i \lambda_{k} t} \phi_{k}(x), \quad a_{k}=\left(a, \phi_{k}\right)_{L^{2}(0,1)}
$$

The inverse data admits the representation:

$$
\left\{r_{0}(t), r_{1}(t)\right\}=\left\{\sum_{k=1}^{\infty} a_{k} e^{i \lambda_{k} t} \phi_{k}^{\prime}(0), \sum_{k=1}^{\infty} a_{k} e^{i \lambda_{k} t} \phi_{k}^{\prime}(1)\right\}
$$


One can prove that $r_{0}, r_{1} \in L^{2}(0, T)$. Using the method from the first section, we recover the eigenvalues $\lambda_{k}$ of $A$ and the products $\phi_{k}^{\prime}(0) a_{k}$ and $\phi_{k}^{\prime}(1) a_{k}$. So (as $a$ is generic) we recovered the spectral data consisting of:

$$
D:=\left\{\lambda_{k}, \frac{\phi_{k}^{\prime}(1)}{\phi_{k}^{\prime}(0)}\right\}_{k=1}^{\infty}
$$

Now from $D$ we construct the spectral function associated with $A$.

Given $\lambda \in \mathbb{C}$, we denote by $y(\cdot, \lambda)$ the solution to:

$$
\left\{\begin{array}{l}
-y^{\prime \prime}(x, \lambda)+q(x) y(x, \lambda)=\lambda y(x, \lambda), \quad 0<x<1, \\
y(0, \lambda)=0, \quad y^{\prime}(0, \lambda)=1
\end{array}\right.
$$

Then, the eigenvalues of the Dirichlet problem of $A$ are exactly the zeros of the function $y(1, \lambda)$, while a family of normalized corresponding eigenfunctions is given by $\phi_{k}(x)=\frac{y\left(x, \lambda_{k}\right)}{\left\|y\left(\cdot, \lambda_{k}\right)\right\|}$. Thus, we can rewrite the second components in $D$ in the following way:

$$
\frac{\phi_{k}^{\prime}(1)}{\phi_{k}^{\prime}(0)}=\frac{y^{\prime}\left(1, \lambda_{k}\right)}{y^{\prime}\left(0, \lambda_{k}\right)}=y^{\prime}\left(1, \lambda_{k}\right)=: A_{k} .
$$

Let us denote by dot the derivative with respect to $\lambda$ and $\lambda_{n}$ be an eigenvalue of $A$. We borrowed the following fact from [17, p. 30]:

$$
\begin{array}{r}
\left\|y\left(\cdot, \lambda_{k}\right)\right\|_{L^{2}}^{2}=y^{\prime}\left(1, \lambda_{k}\right) \dot{y}\left(1, \lambda_{k}\right), \\
y(1, \lambda)=\prod_{n \geqslant 1} \frac{\lambda_{n}-\lambda}{n^{2} \pi^{2}} \\
\dot{y}\left(1, \lambda_{k}\right)=-\frac{1}{k^{2} \pi^{2}} \prod_{n \geqslant 1, n \neq k} \frac{\lambda_{n}-\lambda_{k}}{n^{2} \pi^{2}}=: B_{k} .
\end{array}
$$

Notice that the set of pairs $\left\{\lambda_{k},\left\|y\left(\cdot, \lambda_{k}\right)\right\|_{L^{2}}^{2}\right\}_{k=1}^{\infty}=: \widetilde{D}$ is "classical" spectral data. Using the above relations, we come to $\widetilde{D}=\left\{\lambda_{k}, A_{k} B_{k}\right\}_{k=1}^{\infty}$. Let $\alpha_{k}^{2}:=\left\|y\left(\cdot, \lambda_{k}\right)\right\|_{L^{2}}^{2}=A_{k} B_{k}$, we introduce the spectral function associated with $A$ :

$$
\rho(\lambda)=\left\{\begin{array}{l}
-\sum_{\lambda \leqslant \lambda_{k} \leqslant 0} \frac{1}{\alpha_{k}^{2}} \quad \lambda \leqslant 0, \\
\sum_{0<\lambda_{k} \leqslant \lambda} \frac{1}{\alpha_{k}^{2}} \quad \lambda>0,
\end{array}\right.
$$

which is a monotonously increasing function having jumps at the points of the Dirichlet spectra. The regularized spectral function is introduced by:

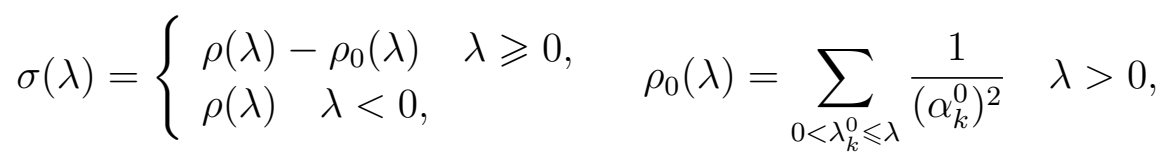

where $\rho_{0}$ is the spectral function associated with the operator $A$ with $q \equiv 0$. The potential can thus be recovered from $\sigma(\lambda)$ by Gelfand-Levitan, Krein or the BC method (see $[6,14]$ ). Once the potential has been found, we can recover the eigenfunctions $\phi_{k}$, the traces $\phi_{k}^{\prime}(0)$ and Fourier coefficients $a_{k}, k=1, \ldots \infty$. Thus, the initial state can be recovered via its Fourier series. 


\subsection{Extension of the inverse data}

We fix $p_{i j} \in C^{1}([0,1] ; \mathbb{C}), d_{1}, d_{2} \in L_{2}(0,1 ; \mathbb{C})$ and consider on interval $(0,1)$ the initial boundary value problem:

$$
\left\{\begin{array}{l}
\frac{\partial}{\partial t}\left(\begin{array}{l}
u \\
v
\end{array}\right)-\frac{\partial}{\partial x}\left(\begin{array}{ll}
0 & 1 \\
1 & 0
\end{array}\right)\left(\begin{array}{l}
u \\
v
\end{array}\right)-\left(\begin{array}{ll}
p_{11} & p_{12} \\
p_{21} & p_{22}
\end{array}\right)\left(\begin{array}{l}
u \\
v
\end{array}\right)=0, \quad t>0 \\
u(0, t)=u(1, t)=0, \quad t>0, \\
\left(\begin{array}{l}
u(x, 0) \\
v(x, 0)
\end{array}\right)=\left(\begin{array}{l}
d_{1}(x) \\
d_{2}(x)
\end{array}\right), \quad 0 \leqslant x \leqslant 1 .
\end{array}\right.
$$

We fix some $T>0$ and define $R(t):=\{v(0, t), v(1, t)\}, 0 \leqslant t \leqslant T$. Here, we focus on the problem of the continuation of the inverse data: we assume that $R(t)$ is known on the interval $(0, T), T>2$, and recover it on the whole real axis. The problem of recovering unknown coefficients $p_{i j}$ and initial state $c_{1,2}$ has been considered in [19,20], where the authors established the uniqueness result, having the response $R(t)$ on the interval $(-T, T)$ for large enough $T$.

We introduce the notations $B=\left(\begin{array}{ll}0 & 1 \\ 1 & 0\end{array}\right), P=\left(\begin{array}{ll}p_{11} & p_{12} \\ p_{21} & p_{22}\end{array}\right), D=\left(\begin{array}{l}d_{1} \\ d_{2}\end{array}\right)$ and the operators $A, A^{*}$ acting by the rule:

$$
\begin{array}{r}
A=B \frac{d}{d x}+P, \quad \text { on }(0,1), \\
A^{*} \psi=-B \frac{d}{d x}+P^{T}, \quad \text { on }(0,1),
\end{array}
$$

with the domains:

$$
D(A)=D\left(A^{*}\right)=\left\{\varphi=\left(\begin{array}{l}
\varphi_{1} \\
\varphi_{2}
\end{array}\right) \in H^{1}\left(0,1 ; \mathbb{C}^{2}\right) \mid \varphi_{1}(0)=\varphi_{1}(1)=0\right\} .
$$

The spectrum of the operator $A$ has the following structure (see [19,20]): $\sigma(A)=\Sigma_{1} \cup \Sigma_{2}$, where $\Sigma_{1} \cap \Sigma_{2}=\emptyset$ and there exists $N_{1} \in \mathbb{N}$ such that

1) $\Sigma_{1}$ consists of $2 N_{1}-1$ eigenvalues including algebraical multiplicities

2) $\Sigma_{2}$ consists of infinite number of eigenvalues of multiplicity one

$3)$ Root vectors of $A$ form a Riesz basis in $L_{2}\left(0,1 ; \mathbb{C}^{2}\right)$.

Let $m$ denote the algebraical multiplicity of eigenvalue $\lambda$, and we introduce the notations:

$$
\begin{gathered}
\Sigma_{1}=\left\{\lambda^{i} \in \sigma(A), m_{i} \geqslant 2,1 \leqslant i \leqslant N\right\}, \\
\Sigma_{2}=\left\{\lambda_{n} \in \sigma(A), \lambda_{n} \text { is simple }, n \in \mathbb{Z}\right\} .
\end{gathered}
$$

Let $e_{1}:=\left(\begin{array}{l}0 \\ 1\end{array}\right)$. The root vectors are introduced in the following way:

$$
\begin{array}{r}
\left(A-\lambda^{i}\right) \phi_{1}^{i}=0, \quad\left(A-\lambda^{i}\right) \phi_{j}^{i}=\phi_{j-1}^{i}, \quad 2 \leqslant j \leqslant m_{i}, \\
\phi_{j}^{i}(0)=e_{1}, \phi_{j}^{i} \in D(A), \quad 1 \leqslant j \leqslant m_{i} .
\end{array}
$$

For the adjoint operator, the following equalities are valid:

$$
\begin{array}{r}
\left(A^{*}-\bar{\lambda}^{i}\right) \psi_{m_{i}}^{i}=0, \quad\left(A^{*}-\bar{\lambda}^{i}\right) \psi_{j}^{i}=\psi_{j+1}^{i}, \quad 1 \leqslant j \leqslant m_{i}-1, \\
\psi_{j}^{i}(0)=e_{1}, \psi_{j}^{i} \in D\left(A^{*}\right), 1 \leqslant j \leqslant m_{i} .
\end{array}
$$


For the simple eigenvalues, we have:

$$
\begin{array}{r}
\left(A-\lambda_{n}\right) \phi_{n}=0, \quad\left(A^{*}-\bar{\lambda}_{n}\right) \psi_{n}=0, \quad \text { for } n \in \mathbb{Z}, \\
\phi_{n}(0)=\psi_{n}(0)=e_{1}, \quad \phi_{n} \in D(A), \quad \psi_{n} \in D\left(A^{*}\right) .
\end{array}
$$

Moreover, the following biorthogonality conditions hold:

$$
\begin{array}{r}
\left(\phi_{j}^{i}, \psi_{n}\right)=0, \quad\left(\phi_{n}, \psi_{j}^{i}\right)=0, \quad\left(\phi_{k}, \psi_{n}\right)=0, \\
\left(\phi_{j}^{i}, \psi_{l}^{k}\right)=0, \quad \text { if } i \neq k \text { or } j \neq l, \\
\rho_{j}^{i}=\left(\phi_{j}^{i}, \psi_{j}^{i}\right), \quad i=1, \ldots, N, \quad j=1, \ldots, m_{i}, \\
\rho_{n}=\left(\phi_{n}, \psi_{n}\right), \quad n \in \mathbb{Z} .
\end{array}
$$

We represent the initial state as the series:

$$
D=\sum_{i=1}^{N} \sum_{j=1}^{m_{i}} d_{j}^{i} \phi_{j}^{i}(x)+\sum_{n \in \mathbb{Z}} d_{n} \phi_{n}(x),
$$

and search for the solution to (4.7) in the form:

$$
\left(\begin{array}{l}
u \\
v
\end{array}\right)(x, t)=\sum_{i=1}^{N} \sum_{j=1}^{m_{i}} c_{j}^{i}(t) \phi_{j}^{i}(x)+\sum_{n \in \mathbb{Z}} c_{n}(t) \phi_{n}(x) .
$$

Using the method of moments, we can derive the system of ODe's for $c_{j}^{i}, i \in\{1, \ldots, N\}$, $j \in\left\{1, \ldots, m_{i}\right\}, c_{n}, n \in \mathbb{Z}$, solving which we obtain:

$$
c_{j}^{i}(t)=e^{\lambda^{i} t}\left[d_{j}^{i}+d_{j+1}^{i} t+d_{j+2}^{i} \frac{t^{2}}{2}+\ldots+d_{m_{i}}^{i} \frac{t^{m_{i}-j}}{\left(m_{i}-j\right) !}\right], \quad c_{n}(t)=d_{n} e^{\lambda_{n} t} .
$$

Notice that the response $\{v(0, t), v(1, t)\}$ has a form depicted in (1.1):

$$
\begin{aligned}
& v(0, t)=\sum_{i=1}^{N} e^{\lambda^{i} t} a_{i}^{0}(t)+\sum_{n \in \mathbb{Z}} e^{\lambda_{n} t} d_{n}\left(\phi_{n}(0)\right)_{2}, \\
& v(1, t)=\sum_{i=1}^{N} e^{\lambda^{i} t} a_{i}^{1}(t)+\sum_{n \in \mathbb{Z}} e^{\lambda_{n} t} d_{n}\left(\phi_{n}(1)\right)_{2},
\end{aligned}
$$

where the coefficients of $a_{i}^{0}(t)=\sum_{k=0}^{m_{i}-1} \alpha_{k}^{i} t^{k}$ are given by

$$
\begin{array}{r}
\alpha_{0}^{i}=\sum_{l=1}^{m_{i}} d_{l}^{i}\left(\phi_{l}^{i}(0)\right)_{2}, \quad \alpha_{1}^{i}=\sum_{l=2}^{m_{i}} d_{l}^{i}\left(\phi_{l-1}^{i}(0)\right)_{2}, \quad \alpha_{2}^{i}=\frac{1}{2} \sum_{l=3}^{m_{i}} d_{l}^{i}\left(\phi_{l-2}^{i}(0)\right)_{2}, \\
\ldots, \alpha_{k}^{i}=\frac{1}{k !} \sum_{l=k+1}^{m_{i}} d_{l}^{i}\left(\phi_{l-k}^{i}(0)\right)_{2}, \ldots \quad \alpha_{m_{i}-1}^{i}=\frac{1}{\left(m_{i}-1\right) !} d_{m_{i}}^{i}\left(\phi_{1}^{i}(0)\right)_{2} .
\end{array}
$$

The coefficients $a_{i}^{1}(t), i=1, \ldots, N$ are defined by the similar formulas.

We assume that the initial state $D$ is generic. Introducing the notation $U:=\left(\begin{array}{l}u \\ v\end{array}\right)$, we consider the dynamical system with the boundary control $f \in L_{2}\left(\mathbb{R}_{+}\right)$:

$$
\left\{\begin{array}{l}
U_{t}-A U=0, \quad 0 \leqslant x \leqslant 1, t>0 \\
u(0, t)=f(t), u(1, t)=0, \quad t>0 \\
U(x, 0)=0
\end{array}\right.
$$

It is not difficult to show that this system is exactly controllable in time $T \geq 2$. This implies (see [5]) that the family $\bigcup_{i=1}^{N}\left\{e^{\lambda^{i} t}, \ldots, t^{m_{i}-1} e^{\lambda^{i} t}\right\} \cup\left\{e^{\lambda_{n} t}\right\}_{n \in \mathbb{Z}}$ forms a Riesz basis in a closure 
of its linear span in $L_{2}((0, T) ; \mathbb{C})$. So we can apply the method from the second sections to recover $\lambda^{i}, m_{i}$, coefficients of polynomials $a_{i}^{0,1}(t) i=1, \ldots, N, \lambda_{n}, n \in \mathbb{Z}$. The latter allows one to extend the inverse data $R(t)$ to all values of $t \in \mathbb{R}$ by formulas (4.9), (4.10). This is important for the solution of the identification problem, see [20].

\section{Acknowledgments}

Sergei Avdonin was supported by the NSF grant DMS 1411564; Alexander Mikhaylov was supported by NSh-1771.2014.1, RFBR 14-01-00306, IRSES (FP7-PEOPLE-2012-IRSES Marie Curie Actions); Victor Mikhaylov was supported by RFBR 14-01-00535, RFBR 14-0131388 and NIR SPbGU 11.38.263.2014.

\section{References}

[1] Avdonin S., Bulanova A. Boundary control approach to the spectral estimation problem. The case of multiple poles. Math. Contr. Sign. Syst., 22 (3), P. 245-265 (2011).

[2] Avdonin S., Bulanova A., Nicolsky D.. Boundary control approach to the spectral estimation problem. The case of simple poles. Sampling Theory in Signal and Image Processing, 8 (3), P. 225-248 (2009).

[3] Avdonin S.A., Belishev M.I. Boundary control and dynamical inverse problem for nonselfadjoint SturmLiouville operator. Control Cybernetics, 26, P. 429-440 (1996).

[4] Avdonin S., Gesztesy F., Makarov K.A. Spectral estimation and inverse initial boundary value problems. Inverse Probl. Imaging, 4 (1), P. 1-9 (2010).

[5] Avdonin S., Ivanov S. Families of exponentials. Cambridge University Press, Cambridge, 1995.

[6] Avdonin S., Mikhaylov V. The boundary control approach to inverse spectral theory. Inverse Problems, 26 (4), P. 045009(19) (2010).

[7] Avdonin S., Mikhaylov V. Inverse source problem for the 1-D Schrödinger equation. Zap. Nauchn. Sem. POMI, 393, P. 5-11 (2011).

[8] Avdonin S.A., Mikhaylov V.S. Spectral estimation problem in infinite dimensional spaces. Zap. Nauchn. Sem. POMI, 422, P. 5-18 (2014).

[9] Avdonin S., Mikhaylov V., Ramdani K. Reconstructing the potential for the 1D Schrödinger equation from boundary measurements, IMA Journal of Mathematical Control and Information, 31 (1), P. 137-150 (2014).

[10] Baudouin L., Puel J.-P. Uniqueness and stability in an inverse problem for the Schrödinger equation. Inverse Problems, 18 P. 1537-1554 (2002).

[11] Belishev . Dynamical systems with boundary control: models and characterization of inverse data. Inverse Problems, 17 (4), P. 659-682 (2001).

[12] Belishev M. On a relation between data of dynamic and spectral inverse problems. J. Math. Sci. (N. Y.), 127 (6), P. 2353-2363 (2005).

[13] Belishev M. Recent progress in the boundary control method. Inverse Problems, 23, (2007).

[14] Belishev M.I., Mikhailov V.S. Unified approach to classical equations of inverse problem theory. Journal of Inverse and Ill-Posed Problems, 20 (4), P. 461-488 (2012).

[15] Mikhaylov A.S., Mikhaylov V.S. Equations of the Boundary Control method for the inverse source problem. Zap. Nauch. Sem POMI, 409, P. 121-128 (2012).

[16] Mercado A., Axel Osses A., Lionel R. Carleman inequalities and inverse problems for the Schrödinger equation. C. R. Acad. Sci. Paris, Ser. I, 346, P. 53-58 (2008).

[17] Pö̈chel J., Trubowitz E. Inverse spectral theory. Boston, MA, Academic Press Inc., 1987.

[18] Tucsnak M., Weiss G. Observation and control for operator semigroups. Birkhaüser Advanced Texts: Basler Lehrbucher. Birkhaüser Verlag, Basel, 2009, 483 p.

[19] Trooshin I., Yamamoto M. Riesz basis of root vectors of a non-symmetric system of first-order ordinary differential operators and application to inverse eigenvalue problems. Appl. Anal., 80, (1-2), P. 19-51 (2001).

[20] Trooshin I., Yamamoto M. Identification problem for a one-dimensional vibrating system. Math. Methods Appl. Sci., 28 (17), P. 2037-2059 (2005). 